The Egyptian Journal of Hospital Medicine (July 2020) Vol. 80, Page 608-614

\title{
Nailfold Capillaroscopy Abnormality in Behcet's Disease and Relation to Disease Activity among Egyptian Patients
}

\author{
Shafica Ibrahim, Magdeldin N Ibrahim, Rasha Mahmoud Hammoda* \\ Department of Internal Medicine, Faculty of Medicine, Ain Shams University, Cairo, Egypt \\ *Corresponding Author: Rasha Mahmoud Hammoda, E-Mail: rasha.hammoda80@gmail.com,
}

Mobile: (+20)01148904211

\begin{abstract}
Background: Behcet's disease is a systemic autoimmune disease. Treatment mainly is immunosuppression. It has no sure diagnostic tests and relies mainly on clinical diagnosis. There are few scores to assess Behcet's disease activity; (Behcet's Disease Current Activity Form) BDCAF score. Nailfold capillaroscopy is a technique to visualize capillaries in nailfold area. It can correlate many diseases with the abnormalities in shape of blood capillaries.
\end{abstract}

Objectives: The aim of the work was to find out nailfold capillaroscopy abnormality in Behcet's disease and its relation to the disease activity.

Patients and Methods: A cross sectional-case control study which was done on 50 Behcet's patients and 30 healthy controls. We assessed nailfold capillaroscopic changes in Behcet's patients in comparison to controls and the correlation of BDCAF score with nailfold capillary abnormalities.

Results: We found that $70 \%$ of Behcet's disease patients had nailfold capillaroscopy abnormalities, a highly statistically significant changes in the capillaries in the form of capillary dilatation (24\%), hemorrhage (54\%), and tortuosity $(64 \%)$ in comparison to the control group $(\mathrm{P}<0.001)$. However, pattern was not specific as BDCAF Score wasn't related to the capillary changes.

Conclusion: Nailfold capillaroscopy showed nonspecific pattern in $70 \%$ Behcet's disease patients. Tortuosity, hemorrhages, dilation were not related to disease activity. Capillaroscopy is a good primary test for Bechet's disease and can reflect the presence and extent of microvascular involvement and thereby might have diagnostic and prognostic value.

Keywords: Behcet's Disease, Nailfold Capillaroscopy Abnormality.

\section{INTRODUCTION}

Behçet's disease (BD) is a systemic variable sized vasculitis of uncertain etiology with specific affection of venules. Onset is typically in young adults with recurrent oral and genital ulceration, uveitis, skin manifestations, arthritis, neurological manifestation, and increased risk of thrombosis. International diagnostic criteria have been proposed; however, diagnosis can be difficult, particularly if the typical ulcers are not clear at presentation. Treatment is challenging and must be tailored to the pattern of organ affection for each patient and often requires combination therapies ${ }^{(\mathbf{1})}$.
BD still remains a clinical diagnosis, and for the purposes of international research the diagnostic criteria proposed by the International Study Group in 1990 and revised in 2010 is now widely accepted ${ }^{(2)}$. Vascular involvement, is one of the serious manifestations of $\mathrm{BD}$, it is considered as a poor prognostic manifestation. Most of the studies about vascular involvement are related to large vessel involvement; however, few studies commented on microvascular damage in BD. Capillaroscopy is a non-invasive diagnostic technique designed to evaluate small vessels of the microcirculation ${ }^{(3)}$. 
$\mathrm{BD}$ is classified as a variable sized vasculitis, and it is characterized by endothelial dysfunction and chronic inflammation. Vascular involvement, which is reported in $18.2 \%$ of the patients, includes arterial and venous involvement. Arterial thrombosis, aneurysms, deep vein thrombosis, large vein thrombosis, and superficial phlebitis are the most common forms of vascular involvement; nevertheless, all sizes of vessels may be involved therefore, the involvement of the capillaries is an expected finding in $\mathrm{BD}^{(3)}$.

The aim of the current work was to find out nailfold capillaroscopy abnormality in Behçet's disease and its relation to the disease activity.

\section{PATIENTS AND METHODS}

This is a cross sectional-case control study which was done on 50 Behcet's disease patients and 30 healthy controls within the year 2018. The study group was divided into 2 groups: (1) 50 patients with Behcet's disease fulfilling the criteria for the diagnosis by International Team for the Revision of the International Criteria for Bechet's Disease ${ }^{(2)}$, patients with other autoimmune diseases, diabetic, hypertensive were excluded from the study. (2) 30 healthy controls who were medically free.

\section{Ethical approval:}

An approval of the study was obtained from Ain Shams University academic and ethical committee. Every patient signed an informed written consent for acceptance of the operation.

Collected data included: Clinical assessment through: Full History taking emphasizing on Behcet's disease clinical data. Thorough clinical examination including general and musculoskeletal system, with special attention to: Recurrent oral, genital ulceration, eye lesions, hemoptysis, CNS manifestations, joint manifestation, laboratory assessment; Complete blood count (CBC), erythrocyte sedimentation rate (ESR) in the first hour, C-reactive protein (CRP), disease activity which was assessed according to BDCAF score (Behcet's Disease Current Activity Form) ${ }^{(\mathbf{4})}$.
Assessment of any activity regarding the eyes using fundus examination, which was done at Ain Shams University Ophthalmology Clinic, focusing on any sign of uveitis, flare cells, retinitis and any other abnormality that could be detected. Assessment of the capillary circulation for patients and control using the nailfold capillaroscopy, which was done using (Optilia Digital Capillaroscopy, Basic kit). The following parameters were considered; Comment on the shape of the capillaries (normal hairpin, or abnormal shapes e.g. Branching capillaries or tortuous), the diameter of the capillaries (dilated loops if the diameter is $>20 \mu \mathrm{m}$ and mega capillaries if $\geq 50 \mu \mathrm{m}$ ), the capillary length (normal or elongated $\geq 300 \mu \mathrm{m}$ ), capillary hemorrhage (two or more in at least two finger ${ }^{(5)}$.

\section{Statistical analysis}

The collected data were coded, processed and analyzed using the SPSS (Statistical Package for the Social Sciences) version 22 for Windows ${ }^{\circledR}$ (IBM SPSS Inc, Chicago, IL, USA). Data were tested for normal distribution using the Shapiro Wilk test. Qualitative data were represented as frequencies and relative percentages. Chi square test $\left(\chi^{2}\right)$ or Fisher's exact test was used to calculate difference between two or more groups of qualitative variables. Quantitative data were expressed as mean \pm SD (Standard deviation) and range. Independent samples t-test was used to compare between two independent groups of normally distributed variables (parametric data). $\mathrm{P}$ value $<0.05$ was considered significant.

\section{RESULTS}

Our BD patients: $62 \%$ (31) were males and $38 \%$ (19) were females, their age ranged between 19-57 years with a mean (32.48 \pm 8.57) years, Control subjects: $50 \%$ (15) were males and $50 \%$ (15) were females, their age ranged between 19-56 years with a mean $(30.367 \pm 10.972)$ years. Regarding BDCAF score findings among patients headache was the most common one with $54 \%$ of patients was affected, , Patients BDCAF score ranged from 0-11 with a mean of 4.9 (Figure 1). 


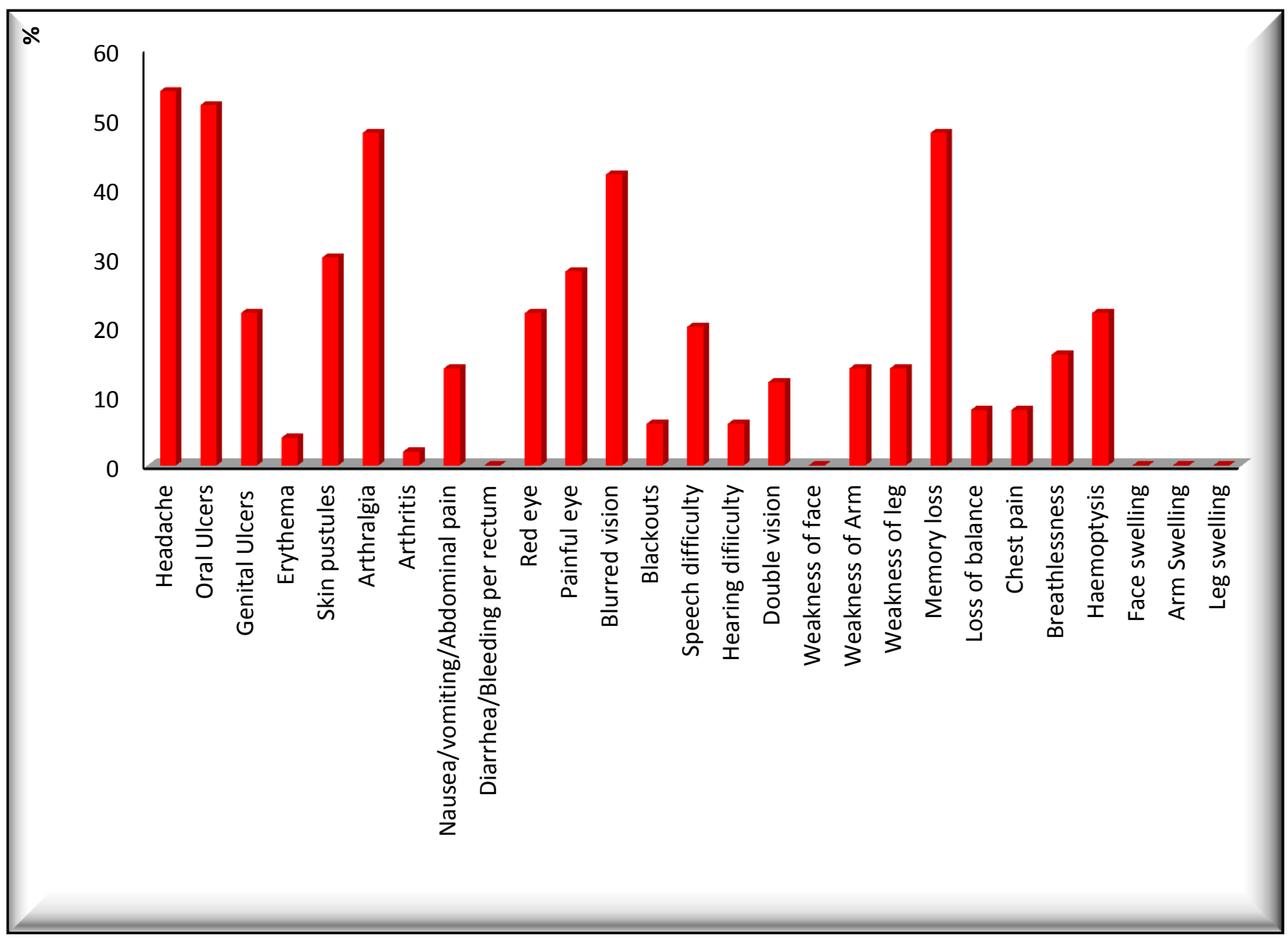

Fig. (1): Distribution of the most common symptoms according to BDCAF score

According to (NFC) abnormalities among patients; capillary hemorrhage, ramified, tortuous capillaries were present in (64\%) of the patients, elongated capillaries in (60\%), and dilated capillaries in (24\%) (Table 1).

Table (1): Nailfold capillaroscopy findings in BD patients

\begin{tabular}{|l|c|c|}
\hline \multirow{2}{*}{\multicolumn{1}{c|}{$($ NFC) findings }} & \multicolumn{2}{c|}{ Patients N=50 } \\
\cline { 2 - 3 } & No. & $24 \%$ \\
\hline Capillary dilatation & 12 & $64 \%$ \\
\hline Capillary hemorrhage & 32 & $64 \%$ \\
\hline Ramified, tortuous capillaries & 32 & $60 \%$ \\
\hline Elongated capillaries & 30 & $0 \%$ \\
\hline Sub-papillary venous plexus & 0 & $0 \%$ \\
\hline Avascular area & 0 & \\
\hline
\end{tabular}

Dilated, tortuous capillaries and presence of capillary hemorrhage were significantly more frequent among patients (Table 2) and mean capillary length and width were significantly higher among patients (Table 3 ). 
Table (2): Comparison between patients and control group regarding nailfold capillary changes

\begin{tabular}{|c|c|c|c|c|c|c|c|c|c|}
\hline \multirow{2}{*}{\multicolumn{2}{|c|}{ Capillaries Changes }} & \multicolumn{6}{|c|}{ Groups } & \multirow{2}{*}{\multicolumn{2}{|c|}{ Chi-Square }} \\
\hline & & \multicolumn{2}{|c|}{ Patients $(\mathrm{N}=50)$} & \multicolumn{2}{|c|}{ Control N=30 } & \multicolumn{2}{|c|}{ Total } & & \\
\hline & & $\mathbf{N}$ & $\%$ & $\mathbf{N}$ & $\%$ & $\mathbf{N}$ & $\%$ & $\mathbf{X}^{2}$ & P-value \\
\hline \multirow{2}{*}{ Capillary dilation } & Positive & 12 & 24 & 0 & 0 & 12 & 15 & \multirow{2}{*}{8.471} & \multirow{2}{*}{$0.004 *$} \\
\hline & Negative & 38 & 76 & 30 & 100 & 68 & 85 & & \\
\hline \multirow{2}{*}{$\begin{array}{l}\text { Capillary } \\
\text { microhemorrhage }\end{array}$} & Positive & 27 & 54 & 0 & 0 & 27 & 33.75 & \multirow{2}{*}{24.453} & \multirow{2}{*}{$<0.001 *$} \\
\hline & Negative & 23 & 46 & 30 & 100 & 53 & 66.25 & & \\
\hline \multirow{2}{*}{$\begin{array}{l}\text { Ramified, tortuous } \\
\text { capillary }\end{array}$} & Positive & 32 & 64 & 0 & 0 & 32 & 40 & \multirow{2}{*}{32.000} & \multirow{2}{*}{$<0.001 *$} \\
\hline & Negative & 18 & 36 & 30 & 100 & 48 & 60 & & \\
\hline
\end{tabular}

Table (3): Comparison between patients and control regarding capillary length and width

\begin{tabular}{|l|c|c|c|}
\hline \multirow{2}{*}{} & \multicolumn{2}{|c|}{ Groups } & \multirow{2}{*}{ P-value } \\
\cline { 2 - 3 } & Patients N=50 & Control N=30 & \multirow{2}{*}{$\begin{array}{c}\text { Capillary length: } \\
\text { Range }\end{array}$} \\
Mean \pm SD & $100-250$ & $110-2001^{*}$ \\
\hline $\begin{array}{l}\text { Capillary width: } \\
\text { Range }\end{array}$ & $167.100 \pm 35.370$ & $123.333 \pm 31.598$ & \\
Mean \pm SD & $20-50$ & $20-25$ & $0.053^{*}$ \\
\hline
\end{tabular}

Behçet's patients whose capillaroscopy showed presence of dilated capillaries, presence of ramified or tortuous capillaries had significantly higher mean values of ESR compared to those patients whose capillaroscopy didn't show these finding. However mean CRP titer was not significantly different (Table 4).

Table (4): Association of NFC abnormalities and ESR and CRP

\begin{tabular}{|c|c|c|c|c|c|c|}
\hline \multirow{2}{*}{\multicolumn{2}{|c|}{ Capillary changes }} & \multicolumn{4}{|c|}{ ESR } & \multirow{2}{*}{ P-value } \\
\hline & & \multirow{2}{*}{$\begin{array}{l}\mathbf{N} \\
12\end{array}$} & \multirow{2}{*}{$\frac{\text { Mean }}{29.750}$} & \multirow{2}{*}{$\begin{array}{l} \pm \\
\pm\end{array}$} & \multirow{2}{*}{$\frac{\text { SD }}{22.145}$} & \\
\hline \multirow{2}{*}{ Capillary dilation } & Positive & & & & & \multirow{2}{*}{$0.053 *$} \\
\hline & Negative & 38 & 15.684 & \pm & 10.086 & \\
\hline \multirow{2}{*}{ Capillary microhemorrhage } & Positive & 27 & 23.963 & \pm & 11.012 & \multirow{2}{*}{0.558} \\
\hline & Negative & 23 & 21.565 & \pm & 17.430 & \\
\hline \multirow{2}{*}{ Capillary ramified, tortuous } & Positive & 32 & 26.469 & \pm & 16.278 & \multirow{2}{*}{$0.015^{*}$} \\
\hline & Negative & 18 & 16.444 & \pm & 5.617 & \\
\hline & & \multicolumn{4}{|c|}{ CRP } & \\
\hline \multirow{2}{*}{ Capillary dilation } & Positive & 12 & 5.667 & \pm & 1.155 & \multirow{2}{*}{0.156} \\
\hline & Negative & 38 & 7.105 & \pm & 3.376 & \\
\hline \multirow{2}{*}{ Capillary microhemorrhage } & Positive & 27 & 6.296 & \pm & 1.815 & \multirow{2}{*}{0.248} \\
\hline & Negative & 23 & 7.304 & \pm & 4.028 & \\
\hline \multirow{2}{*}{ Capillary ramified, tortuous } & Positive & 32 & 6.063 & \pm & 1.294 & \multirow{2}{*}{0.062} \\
\hline & Negative & 18 & 7.711 & \pm & 4.602 & \\
\hline
\end{tabular}

Regarding different Behçet's disease clinical manifestations and nailfold capillaroscopic abnormalities; patients with posterior chamber affection had significantly more frequent presence of ramified and tortuous capillaries compared to those without affection (Table 5) and those with anterior chamber affection had significantly higher mean capillary length (Table 6). 
In addition patients with CNS manifestation more frequently showed ramified and tortuous, dilated capillaries and patients with hemoptysis more frequently showed capillary hemorrhage and ramified, tortuous capillaries (Table 5).

Table (5): Association between NFC abnormalities and clinical manifestations

\begin{tabular}{|c|c|c|c|c|c|c|c|c|c|c|c|}
\hline \multirow{3}{*}{$\begin{array}{l}\text { Clinical } \\
\text { manifestation }\end{array}$} & & & \multicolumn{6}{|c|}{ Nailfold capillary changes } & \multirow{2}{*}{\multicolumn{3}{|c|}{$\begin{array}{c}\text { Chi-square test } \\
\quad \text { (p-value) }\end{array}$}} \\
\hline & & & \multicolumn{2}{|c|}{ Capillary dilation } & \multicolumn{2}{|c|}{$\begin{array}{c}\text { Capillary } \\
\text { microhemorrhage }\end{array}$} & \multicolumn{2}{|c|}{$\begin{array}{c}\text { Capillary } \\
\text { ramified, tortuous }\end{array}$} & & & \\
\hline & & & $\begin{array}{c}\text { Positive } \\
(\mathrm{n}=12)\end{array}$ & $\begin{array}{c}\text { Negative } \\
(\mathrm{n}=38)\end{array}$ & $\begin{array}{c}\text { Positive } \\
(\mathrm{n}=27)\end{array}$ & $\begin{array}{c}\text { Negative } \\
(\mathbf{n}=23)\end{array}$ & $\begin{array}{c}\text { Positive } \\
(\mathrm{n}=32)\end{array}$ & $\begin{array}{c}\text { Negative } \\
(\mathrm{n}=18)\end{array}$ & P1 & $\mathbf{P 2}$ & $\mathbf{P 3}$ \\
\hline \multirow{4}{*}{$\begin{array}{l}\text { Anterior } \\
\text { Chamber } \\
\text { Affection }\end{array}$} & Positive & No. & 4 & 8 & 7 & 5 & 8 & 4 & \multirow{4}{*}{0.385} & \multirow{4}{*}{0.73} & \multirow{4}{*}{0.825} \\
\hline & $(\mathrm{n}=12)$ & $\%$ & $33.3 \%$ & $66.7 \%$ & $58.3 \%$ & $41.7 \%$ & $66.7 \%$ & $33.3 \%$ & & & \\
\hline & Negative & eNo. & 8 & 30 & 20 & 18 & 24 & 14 & & & \\
\hline & $(\mathrm{n}=38)$ & $\%$ & $21.1 \%$ & $78.9 \%$ & $52.6 \%$ & $47.4 \%$ & $63.2 \%$ & $36.8 \%$ & & & \\
\hline \multirow{4}{*}{$\begin{array}{l}\text { Posterior } \\
\text { Chamber } \\
\text { Affection }\end{array}$} & Positive & No. & 3 & 15 & 9 & 9 & 16 & 2 & \multirow{4}{*}{0.362} & \multirow{4}{*}{0.67} & \multirow{4}{*}{$0.015^{*}$} \\
\hline & $(\mathrm{n}=18)$ & $\%$ & $16.7 \%$ & $83.3 \%$ & $50.0 \%$ & $50.0 \%$ & $88.9 \%$ & $11.1 \%$ & & & \\
\hline & Negative & No. & 9 & 23 & 18 & 14 & 16 & 16 & & & \\
\hline & $(\mathrm{n}=32)$ & $\%$ & $28.1 \%$ & $71.9 \%$ & $56.3 \%$ & $43.8 \%$ & $50.0 \%$ & $50.0 \%$ & & & \\
\hline \multirow{4}{*}{ Genital ulcer } & Positive & No. & 2 & 9 & 6 & 5 & 8 & 3 & \multirow{4}{*}{0.741} & \multirow{4}{*}{0.763} & \multirow{4}{*}{0.744} \\
\hline & $(\mathrm{n}=11)$ & $\%$ & $18.2 \%$ & $81.8 \%$ & $54.5 \%$ & $45.5 \%$ & $72.7 \%$ & $27.3 \%$ & & & \\
\hline & \multirow{2}{*}{$\begin{array}{c}\text { Negative } \\
(\mathrm{n}=39)\end{array}$} & eNo. & 10 & 29 & 21 & 18 & 24 & 15 & & & \\
\hline & & $\%$ & $25.6 \%$ & $74.4 \%$ & $53.8 \%$ & $46.2 \%$ & $61.5 \%$ & $38.5 \%$ & & & \\
\hline \multirow{4}{*}{ Oral ulcer } & \multirow{2}{*}{$\begin{array}{r}\text { Positive } \\
(\mathrm{n}=25)\end{array}$} & No. & 8 & 17 & 16 & 9 & 18 & 7 & \multirow{4}{*}{0.321} & \multirow{4}{*}{0.256} & \multirow{4}{*}{0.377} \\
\hline & & $\%$ & $32.0 \%$ & $68.0 \%$ & $64.0 \%$ & $36.0 \%$ & $72.0 \%$ & $28.0 \%$ & & & \\
\hline & Negative & eNo. & 4 & 21 & 11 & 14 & 14 & 11 & & & \\
\hline & $(\mathrm{n}=25)$ & $\%$ & $16.0 \%$ & $84.0 \%$ & $44.0 \%$ & $56.0 \%$ & $56.0 \%$ & $44.0 \%$ & & & \\
\hline \multirow{4}{*}{$\begin{array}{l}\text { CNS } \\
\text { manifestations } \\
\text { (weakness } \\
\text { upper- } \\
\text { lowerlimb) }\end{array}$} & Positive & No. & 8 & 6 & 12 & 2 & 13 & 1 & \multirow{4}{*}{$0.002 *$} & \multirow{4}{*}{$0.013^{*}$} & \multirow{4}{*}{$0.020^{*}$} \\
\hline & $(\mathrm{n}=14)$ & $\%$ & $57.1 \%$ & $42.9 \%$ & $85.7 \%$ & $14.3 \%$ & $92.9 \%$ & $7.1 \%$ & & & \\
\hline & Negative & No. & 4 & 32 & 15 & 21 & 19 & 17 & & & \\
\hline & $(\mathrm{n}=36)$ & $\%$ & $11.1 \%$ & $88.9 \%$ & $41.7 \%$ & $58.3 \%$ & $52.8 \%$ & $47.2 \%$ & & & \\
\hline & Positive & No. & 6 & 5 & 10 & 1 & 11 & 0 & & & \\
\hline Hemontysis & $(\mathrm{n}=11)$ & $\%$ & $54.5 \%$ & $45.5 \%$ & $90.9 \%$ & $9.1 \%$ & $100.0 \%$ & $0.0 \%$ & 0321 & $<0006 *$ & $<0004 *$ \\
\hline Henoptysis & Negative & No. & 6 & 33 & 17 & 22 & 21 & 18 & 0.021 & 0.000 & 0.004 \\
\hline & $(\mathrm{n}=39)$ & $\%$ & $15.4 \%$ & $84.6 \%$ & $43.6 \%$ & $56.4 \%$ & $53.8 \%$ & $46.2 \%$ & & & \\
\hline & Positive & No. & 5 & 19 & 10 & 14 & 18 & 6 & & & \\
\hline $\mathbf{A r}$ & $(\mathrm{n}=24)$ & $\%$ & $20.8 \%$ & $79.2 \%$ & $41.7 \%$ & $58.3 \%$ & $75.0 \%$ & $25.0 \%$ & 0863 & 0162 & 0207 \\
\hline Artinritis & Negative & No. & 7 & 19 & 17 & 9 & 14 & 12 & 0.803 & 0.102 & 0.201 \\
\hline & $(\mathrm{n}=26)$ & $\%$ & $26.9 \%$ & $73.1 \%$ & $65.4 \%$ & $34.6 \%$ & $53.8 \%$ & $46.2 \%$ & & & \\
\hline
\end{tabular}

P1: Comparison between Capillary dilation with clinical manifestation

P2: Comparison between Capillary microhaemorrhage with clinical manifestation

P3: Comparison between Capillary ramified, tortuous with clinical manifestation 
Table (6): Comparison between patients with and without (anterior-posterior) chamber affection regarding nailfold capillary length and width

\begin{tabular}{|c|c|c|c|c|c|c|c|}
\hline \multirow{3}{*}{ Capillary changes } & \multicolumn{6}{|c|}{ Anterior chamber affection } & \multirow{3}{*}{ P-value } \\
\hline & \multicolumn{3}{|c|}{$\begin{array}{c}\text { Positive } \\
(\mathrm{N}=12)\end{array}$} & \multicolumn{3}{|c|}{$\begin{array}{c}\text { Negative } \\
(\mathrm{N}=38)\end{array}$} & \\
\hline & Mean & \pm & SD & Mean & \pm & SD & \\
\hline Capillary length & 197.750 & \pm & 31.560 & 157.421 & \pm & 30.980 & $<0.001^{*}$ \\
\hline \multirow[t]{2}{*}{ Capillary width } & 30.167 & \pm & 5.734 & 31.711 & \pm & 9.016 & 0.580 \\
\hline & \multicolumn{6}{|c|}{ Posterior chamber affection } & \\
\hline Capillary length & 164.556 & \pm & 32.819 & 168.531 & \pm & 37.160 & 0.707 \\
\hline Capillary width & 30.778 & \pm & 8.454 & 31.656 & \pm & 8.361 & 0.724 \\
\hline
\end{tabular}

Other clinical manifestation didn't show significant difference. On comparing patients with nailfold capillary abnormalities and those without regarding mean BDCAF score, there was no significant statistical difference (Table 7).

Table (7): Comparison between patients with nailfold capillary abnormalities and those without regarding mean BDCAF score

\begin{tabular}{|c|c|c|c|c|c|c|}
\hline \multirow{2}{*}{\multicolumn{2}{|c|}{ Variants }} & \multicolumn{4}{|c|}{ BDCAF score } & \multirow{2}{*}{ P-value } \\
\hline & & \multirow{2}{*}{$\begin{array}{c}\mathbf{N} \\
12\end{array}$} & \multicolumn{3}{|c|}{ Mean \pm SD } & \\
\hline \multirow{2}{*}{ Elongated capillaries } & Positive & & 6.250 & \pm & 3.671 & \multirow{2}{*}{0.134} \\
\hline & Negative & 38 & 4.500 & \pm & 3.407 & \\
\hline \multirow{2}{*}{ Capillary dilation } & Positive & 12 & 6.000 & \pm & 3.717 & \multirow{2}{*}{0.226} \\
\hline & Negative & 38 & 4.579 & \pm & 3.430 & \\
\hline \multirow{2}{*}{$\begin{array}{l}\text { Capillary } \\
\text { microhemorrhage }\end{array}$} & Positive & 27 & 4.778 & \pm & 3.566 & \multirow{2}{*}{0.760} \\
\hline & Negative & 23 & 5.087 & \pm & 3.528 & \\
\hline \multirow{2}{*}{$\begin{array}{l}\text { Capillary ramified, } \\
\text { tortuous }\end{array}$} & Positive & 32 & 4.875 & \pm & 3.892 & \multirow{2}{*}{0.905} \\
\hline & Negative & 18 & 5.000 & \pm & 2.828 & \\
\hline
\end{tabular}




\section{DISCUSSION}

Behcet's disease is a systemic vasculitis, that has an autoimmune etiology but with uncertain mechanisms it may complicates with multiple vascular complication (6). Nailfold videocapillaroscopy (NVC) has been used for the analysis of microvascular abnormalities, which are present in several rheumatic disorders including systemic sclerosis, systemic lupus erythematosus ${ }^{(7)}$.

In the current study the most frequent NFC abnormalities in $\mathrm{BD}$ patients were microhemorrhage in $(64 \%)$, ramified, tortuous capillaries in $(64 \%)$ and elongated capillaries in $(60 \%)$ of patients; coming in agreement with Movasat et al. ${ }^{(8)}$ who demonstrated that $70 \%$ of his Behcet patients had abnormal capillary pattern; most prevalent change was tortuosity $64 \%$, microhemorrhages $54 \%$, and capillary dilation $12 \%$ respectively, also Aytekin et al. ${ }^{\text {(9) }}$ concluded that $75 \%$ of their studied patients showed abnormal nailfold capillary findings in the form of dilated capillaries and presence of capillary hemorrhage, also Alan et $\boldsymbol{a l}^{\left({ }^{(\mathbf{1 0})}\right.}$ reported that tortuous capillaries was present in $91 \%$ of his studied patients.

On comparing our patients and healthy control regarding nailfold capillaroscopic findings, abnormal findings like dilated, ramified, tortuous capillaries and capillary hemorrhage were more frequently observed among patients compared to control. Moreover mean capillary length and width were significantly higher among patients. Our data were nearly similar to Tabanlioglu-Onan et al. ${ }^{(3)}$ who concluded that capillary morphologic alterations were encountered significantly more in BD group, however Wechsler et al. ${ }^{(11)}$ slightly undermined the role of capillaroscopy in Behcet's disease which probably was related to the time of the study.

We observed that BD patients who showed dilated, ramified or tortuous capillaries had significantly high ESR mean values, which was in harmony to Tabanlioglu-Onan et $\boldsymbol{a l} .^{(3)}$ who documented the presence of the relation of acute phase reactant (ESR-CRP) and morphological abnormalities of nailfold capillaroscopy in BD patients.

Several studies suggested that NVC might be useful in the determination of the systemic extension of vascular damage, systemic manifestations and the degree of vessel wall alteration in $\mathrm{BD}^{(3)}$.

In our work regarding study the association of different BD clinical manifestations and nailfoldcapillaroscopic abnormalities, we observed that patients with posterior chamber affection showed more frequent presence of ramified and tortuous capillaries and also those with anterior chamber affection had significantly higher mean capillary length compared to patients without affection. Moreover patients with CNS manifestations showed higher frequency of the presence of capillary hemorrhage, tortuous, ramified and dilated capillaries and patients with hemoptysis more frequently showed capillary hemorrhage and ramified, tortuous capillaries. However BDCAF score was not related to capillaroscopic abnormalities. These were in agreement with Movasat et $\boldsymbol{a l} .{ }^{(8)}$ study who concluded that the presence of capillary hemorrhages tended to be associated with articular symptoms. Also Vaiopoulos et al. (12) reported a significant association between capillaroscopic abnormalities and skin manifestations, arthritis/arthralgia, and pathergy test in their patients. However, Aytekin et al. ${ }^{(9)}$ reported that capillaroscopic changes were not associated with clinical characteristics. Also Alan et al. ${ }^{(\mathbf{1 0 )}}$ did not find any association between abnormal capillary changes and different manifestations of BD.

In conclusion nailfold capillary abnormalities were more frequently present in patients with $\mathrm{BD}$ compared to healthy control, some clinical manifestations were associated with some (NFC) abnormalities, however (NFC) abnormalities weren't related to disease activity. Further studies are needed to identify the diagnostic and prognostic value of capillaroscopy in $\mathrm{BD}$. 


\section{REFERENCES}

1. Kontogiannis V, Powell R (2000): Behçet's disease. Postgraduate Medical Journal, 76:629637.

2. International Team for the Revision of the International Criteria for Behçet's Disease (2014): The International Criteria for Behçet's Disease (ICBD), a collaborative study of 27 countries on the sensitivity and specificity of the new criteria. J Eur Acad Dermatol Venereol., 28:338-347.

3. Tabanlioglu-Onan $D$, İncel-Uysal $P$, Hayran Y et al. (2019): Dermatoscopic assessment of nailfold capillary abnormalities in Behçet's disease and correlation of the findings with disease activity and severity. Dermatologica Sinica., 37(1): 40-45.

4. Bhakta B, Brennan P, James T et al. (1999): Behc $\square$ et's disease: evaluation of a new instrument to measure clinical activity. Rheumatology, 38(8): 728-733.

5. Chojnowski M, Felis-Giemza A, Olesińska M (2016): Capillaroscopy - a role in modern rheumatology. Reumatologia, 2: 67-72.

6. Zeidan MJ, Saadoun D, Garrido $M$ et al. (2016): Behçet's disease physiopathology: a contemporary review. Auto Immun Highlights, 7(1):4.

7. Chojnowski M, Felis-Giemza A, Olesińska M et al. (2016): Capillaroscopy - a role in modern rheumatology. Reumatologia, 54(2):67-72.

8. Movasat A, Shahram F, Carreira $P$ et al. (2009): Nailfold capillaroscopy in Behçet's disease, analysis of 128 patients. Clin Rheumatol., 28(5):603-605.

9. Aytekin S, Yuksel E, Aydin F et al. (2014): Nailfold capillaroscopy in Behçet disease, performed using video dermoscopy. Clin Exp Dermatol., 39(4):443-447.

10. Alan S, Balkarlı A, Tuna S et al. (2016): The nailfold video capillaroscopy findings of Behçet's syndrome. Dermatologica Sinica., 34(2): 74-77.

11. Wechsler B, Le T, Mouthon J et al. (1984): Periungual capillaroscopic aspects in Behçet's disease. Apropos of 30 cases. Ann Dermatol Venereol., 111(6-7):543-50.

12. Aiopoulos G, Pangratis N, Samarkos M et al. (2005): Nailfold capillary abnormalities in Behcet's disease. J Rheumatol., 22: 1108-1111. 